\title{
Noncommutative real scalar field theory in $2+1$ dimensions at finite temperature
}

\section{C.D. Fosco}

Centro Atómico Bariloche and Instituto Balseiro, Comisión Nacional de Energía Atómica, R8402AGP Bariloche, Argentina E-mail: fosco@cab.cnea.gov.ar

\section{G.A. Silva}

IFLP/CONICET-Departamento de Física, Universidad Nacional de La Plata, C.C. 67, 1900 La Plata, Argentina

E-mail: silva@fisica.unlp.edu.ar

ABSTRACT: We study thermal effects for a noncommutative real scalar field in $2+1$ dimensions including a Grosse-Wulkenhaar term. Using a perturbative expansion for the free energy, we deduce some general properties of the corresponding contributions, in the thermodynamic limit. We show that the model can be consistently interpreted as defined on a finite volume, which is naturally determined by the noncommutativity scale.

KEYwords: Field Theories in Lower Dimensions, Non-Commutative Geometry, Thermal Field Theory. 


\section{Contents}

1. Introduction 1

2. Perturbative calculation of the free energy 3

2.1 General considerations 3

2.2 The model

2.3 Zeroth order ('ideal gas')

2.3.1 The self-dual $\Omega^{2}=1$ case

2.3.2 The general case 8

2.4 First-order term 10

2.4 .1 Self-dual case 10

2.4.2 General case 14

2.5 Summation of the planar ring diagrams for the self-dual case

3. Conclusions

A. Useful formulae and Feynman diagrams conventions 16

\section{Introduction}

The class of Noncommutative Quantum Field Theories (NCQFT's) obtained by a Moyal deformation of the usual (pointwise) product of functions, has been extensively studied in recent years [1], 2]. One of the main reasons for the renewed interest in this subject may be found in its relevance to the dynamics of open string theories in non-vanishing constant antisymmetric NS backgrounds, a set up that leads to noncommutativity between the string endpoint coordinates [3].

From a quite different standpoint, this topic has also attracted attention because NCQFT's seem to be very good candidates for an effective description of the Quantum Hall Effect (QHE) ॠ. Indeed, the existence of a strong magnetic field normal to an (essentially) two-dimensional sample, paves the way to the use of the Peierls substitution, whereby the two spatial coordinates corresponding to each charged particle become noncommuting operators [5].

In these NCQFT's one usually considers quantum fields endowed with a noncommutative (Moyal) product which, for two functions $f(x)$ and $g(x)\left(x \in \mathbb{R}^{(d)}\right)$, may be defined as follows:

$$
(f \star g)(x) \equiv\left[e^{\frac{i}{2} \theta_{\mu \nu} \partial_{\mu}^{x} \partial_{\nu}^{y}} f(x) g(y)\right]_{y \rightarrow x} .
$$

Here, $\theta_{\mu \nu}$ is a constant antisymmetric tensor, and $\mu, \nu$ run over the spacetime indices. Since we shall be concerned with the case of $2+1$-dimensional, i.e., 'planar' theories, the $\theta_{\mu \nu}$ 
tensor does necessarily have a zero mode. We shall assume that this zero mode corresponds to the time-like $(\mu=0)$ direction, having in mind instances where the noncommutativity is, indeed, due to a physical magnetic field, as in the QHE. The $\theta_{\mu \nu}$ tensor will thus verify $\theta_{0 \mu}=0$ and the time-like coordinate $x_{0}$ behaves, to all effects, as a commuting object. ${ }^{1}$

In this paper, we shall be concerned with the calculation of thermal effects in the NCQFT of a real scalar field. Many interesting results have recently been obtained by considering noncommutative systems obtained by a Moyal deformation of a standard QFT, at a finite temperature [6-9]. However, we want to consider here the case of a model equipped with a Grosse-Wulkenhaar (GW) term [10, 11] (see also [12]). The reason for considering this kind of theory, rather than standard ones is, in our context, twofold. Firstly, from a fundamental QFT point of view (not necessarily at a finite temperature), we should do that in order to solve the non-decoupling of UV and IR fluctuations that unavoidably manifests in the absence of such a term (a phenomenon usually known as 'IR-UV mixing' [13]). As a by product, one finds that a NCQFT without a GW-term is, moreover, 'anomalous' under the Langmann-Szabo duality [14].

Secondly, and this is particularly relevant for the case at hand, one may also want to include the GW-term because it effectively confines the system to a finite volume, which is controlled by the strength of that term. This situation is, indeed, of physical interest when one wants to consider NCQFT at a finite temperature, in particular for the calculation of the free energy. There, one has to assume that the theory has been defined on a finite volume, and that volume tends to infinity at the end, when one takes the thermodynamic limit. This is not an issue in the commutative case, where one can simply use a box and impose periodic boundary conditions for the fields. The situation is not so simple in the noncommutative case, however, since one should have to face the problem of imposing boundary conditions for the NCQFT defined in a finite volume, with the related technical difficulties.

The GW-term introduces, for the case at hand, a kind of external harmonic potential. It should be reminded that noncommutative theories including a coupling to an external (usually magnetic) field do have interesting properties, even in the context of noncommutative quantum mechanics [15, 16]. Indeed, for a (noncommutative) charged particle an interesting distinction between two different phases naturally emerges, depending on the ratio between the external magnetic field strength $B$ and the noncommutativity parameter $\theta$. Those phases, corresponding to Hamiltonians having essentially different spectra, are separated by a critical point determined by a relation $\theta B=\kappa$, where $\kappa$ is a numerical constant of order 1 , whose precise value depends on the units and conventions adopted. At that critical point the system becomes exactly solvable [17] (even in the presence of an external potential [16]) and there is, moreover, an effective 'dimensional reduction' 15].

For the real scalar field we shall deal with in this work, there is also an interesting interplay between the strength of the GW-term $\Omega^{2}$ and the noncommutativity parameter $\theta$, although the symmetry properties are quite different to the ones in the charged field case.

In this paper we study the perturbative calculation of the free energy for that model,

\footnotetext{
${ }^{1}$ This is particularly relevant to thermal field theory since the temperature is a commuting object so must therefore be the imaginary time $\tau$.
} 
focussing on the general properties of the first few terms, providing explicit results whenever possible. We do that mostly for the self-dual case $(\Omega=1)$, and discuss the relation between them with the $\Omega \neq 1$ case. The article is organized as follows: in section 2 , we present the perturbative calculation of the free energy for a real scalar field in $2+1$ dimensions with a Grosse-Wulkenhaar term. In section 3, we present our conclusions.

\section{Perturbative calculation of the free energy}

\subsection{General considerations}

Let us briefly review here the usual approach to the calculation of the free energy, in the path-integral (imaginary-time) context [18], to apply it afterwards to the real scalar field case.

We shall start from the partition function $\mathcal{Z}$, depending on $\beta \equiv T^{-1}$ and, eventually (i.e., when there is a non-trivial internal conserved charge) on a chemical potential. The functional integral representation of $\mathcal{Z}$ has the form

$$
\mathcal{Z}=\int \mathcal{D} \mu e^{-S}
$$

where $\mathcal{D} \mu$ denotes the functional integration measure corresponding to the space of fields being considered, which requires periodic or antiperiodic conditions for the imaginary-time coordinate $x_{0} \equiv \tau$, according to the fields being bosonic or fermionic, respectively.

In (2.1) $S$ denotes the Euclidean action for a finite imaginary-time interval, namely, $\tau \in[0, \beta]$. The actions that we shall consider may be naturally decomposed as follows:

$$
S=S_{0}+S_{I}
$$

where $S_{0}$ denotes the free, i.e., quadratic, part of the action, and $S_{I}$ the interaction piece (at least cubic in the fields). Note that the GW-term shall be thus included in $S_{0}$.

Based on the above decomposition for the action, one arrives to the expression

$$
\mathcal{Z}=\mathcal{Z}_{0}\left\langle e^{-S_{I}}\right\rangle
$$

where

$$
\mathcal{Z}_{0}=\int \mathcal{D} \mu e^{-S_{0}}
$$

and we have introduced the notation:

$$
\langle\ldots\rangle \equiv \frac{1}{\mathcal{Z}_{0}} \int \mathcal{D} \mu \ldots e^{-S_{0}},
$$

for Gaussian averages defined by the free action.

When constructing an expansion in powers of $S_{I}$, it turns out to be simpler to consider the free energy $\mathcal{F} \equiv-\frac{1}{\beta} \ln \mathcal{Z}$. Indeed, one easily finds that

$$
\mathcal{F}=\mathcal{F}_{0}+\mathcal{F}_{I}
$$


where

$$
\mathcal{F}_{0}=-\frac{1}{\beta} \ln \mathcal{Z}_{0}
$$

and

$$
\mathcal{F}_{I} \equiv-\frac{1}{\beta} \ln \left\langle e^{-S_{I}}\right\rangle
$$

Expanding $\mathcal{F}_{I}$ in powers of $S_{I}$, one obtains

$$
\mathcal{F}_{I}=\mathcal{F}_{I}^{(1)}+\mathcal{F}_{I}^{(2)}+\mathcal{F}_{I}^{(3)}+\ldots
$$

where

$$
\begin{gathered}
\mathcal{F}_{I}^{(1)}=\frac{1}{\beta}\left\langle S_{I}\right\rangle, \\
\mathcal{F}_{I}^{(2)}=-\frac{1}{2 ! \beta}\left\langle\left(S_{I}-\left\langle S_{I}\right\rangle\right)^{2}\right\rangle, \\
\mathcal{F}_{I}^{(3)}=\frac{1}{3 ! \beta}\left\langle\left(S_{I}-\left\langle S_{I}\right\rangle\right)^{3}\right\rangle, \ldots
\end{gathered}
$$

In the remaining parts of this section, we first define the model that we shall study in detail, and afterwards we calculate the first few terms in the above-defined expansion.

\subsection{The model}

The model is defined by an Euclidean action $S=S_{0}+S_{I}$, with

$$
S_{0}=\frac{1}{2} \int_{0}^{\beta} d \tau \int d^{2} x\left[\partial_{\mu} \phi \star \partial_{\mu} \phi+\Omega^{2}\left(\tilde{x}_{i} \phi\right) \star\left(\tilde{x}_{i} \phi\right)+m^{2} \phi \star \phi\right]
$$

where $\tilde{x}_{i} \equiv 2\left(\theta^{-1}\right)_{i j} x_{j}$, and

$$
S_{I}=\frac{\lambda}{4 !} \int_{0}^{\beta} d \tau \int d^{2} x \phi \star \phi \star \phi \star \phi .
$$

We shall assume that $\theta>0$ and $\Omega \geq 0$ (without any lose of generality).

The harmonic potential proportional to $\Omega^{2}$ in 2.13 is the GW term. It has been shown that its confining properties provide an infrared cutoff and, by the same token, tame the IR problem due to IR/UV mixing [11.

By using elementary properties of the Moyal product, the free action $S_{0}$ may also be written in the equivalent way

$$
\begin{aligned}
S_{0}=\frac{1}{2} \int_{0}^{\beta} d \tau \int d^{2} x & {\left[\partial_{\tau} \phi \star \partial_{\tau} \phi+\left(\frac{1+\Omega^{2}}{2}\right) \phi \star \tilde{x}_{j} \star \tilde{x}_{j} \star \phi\right.} \\
& \left.-\left(\frac{1-\Omega^{2}}{2}\right)\left(\tilde{x}_{j} \star \phi \star \tilde{x}_{j} \star \phi\right)+m^{2} \phi \star \phi\right] .
\end{aligned}
$$




\subsection{Zeroth order ('ideal gas')}

The zeroth-order term $\mathcal{Z}_{0}$ is obtained from the evaluation of a Gaussian functional integral,

$$
\mathcal{Z}_{0}=\int \mathcal{D} \phi e^{-S_{0}[\phi]} .
$$

Following the usual procedure of QFT at finite temperature, we decompose the field $\phi$, periodic in the time coordinate, in terms of its Fourier components

$$
\phi(x)=\phi(\tau, \mathbf{x})=\beta^{-\frac{1}{2}} \sum_{n=-\infty}^{+\infty} e^{i \omega_{n} \tau} \phi_{n}(\mathbf{x})
$$

with $\omega_{n}=\frac{2 \pi n}{\beta}$. Since we are considering a real field, $\phi^{\dagger}=\phi$, one has

$$
\phi_{0}^{\dagger}(\mathbf{x})=\phi_{0}(\mathbf{x}), \quad \phi_{n}^{\dagger}(\mathbf{x})=\phi_{-n}(\mathbf{x}), \forall n \geq 1 .
$$

In terms of these modes, the free action becomes a decoupled sum of $(d=2)$ actions, involving a real field $\phi_{0}$ and an infinite number of complex fields $\phi_{n}$

$$
S_{0}[\phi]=S_{0}^{(0)}\left[\phi_{0}\right]+\sum_{n=1}^{\infty} S_{0}^{(n)}\left[\phi_{n}^{\dagger}, \phi_{n}\right]
$$

where

$$
S_{0}^{(0)}\left[\phi_{0}\right]=\frac{1}{2} \int d^{2} x\left[\partial_{j} \phi_{0} \star \partial_{j} \phi_{0}+\Omega^{2}\left(\tilde{x}_{j} \phi_{0}\right) \star\left(\tilde{x}_{j} \phi_{0}\right)+m^{2} \phi_{0} \star \phi_{0}\right]
$$

and

$$
\begin{array}{r}
S_{0}^{(n)}\left[\phi_{n}^{\dagger}, \phi_{n}\right]=\int d^{2} x\left[\partial_{j} \phi_{n}^{\dagger} \star \partial_{j} \phi_{n}+\Omega^{2}\left(\tilde{x}_{j} \phi_{n}^{\dagger}\right) \star\left(\tilde{x}_{j} \phi_{n}\right)\right. \\
\left.+\left(m^{2}+\omega_{n}^{2}\right) \phi_{n}^{\dagger} \star \phi_{n}\right], \quad n \geq 1 .
\end{array}
$$

For each one of these two-dimensional theories we use the matrix base [19] to expand the fields as

$$
\begin{aligned}
& \phi_{n}(\mathbf{x})=\sum_{i, j=0}^{\infty} \phi_{n}^{(i, j)} b^{(i, j)}(\mathbf{x}) \\
& \phi_{n}^{\dagger}(\mathbf{x})=\sum_{i, j=0}^{\infty} \bar{\phi}_{n}^{(i, j)} b^{(j, i)}(\mathbf{x}) .
\end{aligned}
$$

The integration measure $\mathcal{D} \phi$ in $(2.16)$ is defined in terms of the Fourier components (2.17) as

$$
\mathcal{D} \phi=\mathcal{D} \phi_{0} \prod_{n=1}^{\infty} \mathcal{D} \phi_{n}^{\dagger} \mathcal{D} \phi_{n} .
$$

The explicit form for them is

$$
\mathcal{D} \phi_{0}=\left(\prod_{i} \mathcal{D} \phi_{0}^{(i, i)}\right) \prod_{i<j} \mathcal{D} \bar{\phi}_{0}^{(i, j)} \mathcal{D} \phi_{0}^{(i, j)},
$$


and

$$
\mathcal{D} \phi_{n}^{\dagger} \mathcal{D} \phi_{n}=\prod_{i j} \mathcal{D} \bar{\phi}_{n}^{(i, j)} \mathcal{D} \phi_{n}^{(i, j)}
$$

In terms of the matrix base decomposition (2.22), the actions (2.20)-(2.21) take the form

$$
\begin{aligned}
S_{0}^{(0)}\left[\phi_{0}\right] & =(2 \pi \theta) \frac{1}{2} \sum_{i_{1}, i_{2} ; j_{1}, j_{2}} \phi_{0}^{\left(i_{1}, i_{2}\right)} \mathcal{G}_{0}^{\left(i_{2}, i_{1} ; j_{1}, j_{2}\right)} \phi_{0}^{\left(j_{1}, j_{2}\right)} \\
& =(2 \pi \theta) \frac{1}{2} \sum_{i ; j} \phi_{0}^{(i, i)} \mathcal{G}_{0}^{(i, i ; j, j)} \phi_{0}^{(j, j)}+(2 \pi \theta) \sum_{i_{1}<i_{2} ; j_{1}<j_{2}} \bar{\phi}_{0}^{\left(i_{1}, i_{2}\right)} \mathcal{G}_{0}^{\left(i_{1}, i_{2} ; j_{1}, j_{2}\right)} \phi_{0}^{\left(j_{1}, j_{2} \chi_{2}\right.} \chi_{2.26)}
\end{aligned}
$$

and

$$
S_{0}^{(n)}\left[\phi_{n}^{\dagger}, \phi_{n}\right]=2 \pi \theta \sum_{i_{1}, i_{2} ; j_{1}, j_{2}} \bar{\phi}_{n}^{\left(i_{1}, i_{2}\right)} \mathcal{G}_{n}^{\left(i_{1}, i_{2} ; j_{1}, j_{2}\right)} \phi_{n}^{\left(j_{1}, j_{2}\right)}, \quad \forall n \geq 1
$$

where $^{2}$

$$
\begin{aligned}
\mathcal{G}_{n}^{\left(i_{1}, i_{2} ; j_{1}, j_{2}\right)}= & {\left[m^{2}+\omega_{n}^{2}+\mu^{2}\left(i_{1}+i_{2}+1\right)\right] \delta_{i_{1} j_{1}} \delta_{i_{2} j_{2}} } \\
& -\mu^{2} \sqrt{\omega} \sqrt{\left(i_{1}+1\right)\left(i_{2}+1\right)} \delta_{i_{1}+1, j_{1}} \delta_{i_{2}+1, j_{2}} \\
& -\mu^{2} \sqrt{\omega} \sqrt{i_{1} i_{2}} \delta_{i_{1}-1, j_{1}} \delta_{i_{2}-1, j_{2}}
\end{aligned}
$$

with

$$
\mu^{2}=2 \frac{\left(1+\Omega^{2}\right)}{\theta}, \quad \sqrt{\omega}=\frac{1-\Omega^{2}}{1+\Omega^{2}} .
$$

The integrals over the different Fourier modes decouple,

$$
\mathcal{Z}_{0}=\prod_{n=0}^{\infty} \mathcal{Z}_{0}^{(n)}
$$

where

$$
\mathcal{Z}_{0}^{(0)}=\int \mathcal{D} \phi_{0} e^{-S_{0}^{(0)}\left[\phi_{0}\right]}
$$

and

$$
\mathcal{Z}_{0}^{(n)}=\int \mathcal{D} \phi_{n}^{\dagger} \mathcal{D} \phi_{n} e^{-S_{0}^{(n)}\left[\phi_{n}^{\dagger}, \phi_{n}\right]}
$$

A careful use of the matrix base decomposition shows that the following expressions hold true $^{3}$

$$
\ln \mathcal{Z}_{0}^{(0)}=-\frac{1}{2} \operatorname{Tr} \ln \mathcal{G}_{0}
$$

and

$$
\ln \mathcal{Z}_{0}^{(n)}=-\operatorname{Tr} \ln \mathcal{G}_{n}
$$

\footnotetext{
${ }^{2}$ Note that our convention for the kernel differs with the one used in 10, 11 in a transposition of the first pair of indices.

${ }^{3}$ Although we are taking the logarithm of a dimensional quantity in 2.35), a precise meaning can be given to the formula using the analytic regularization technique [20].
} 
In this last expressions the trace operation $\operatorname{Tr}$ should be understood as taken over the whole set of matrix base elements $\left\{b^{(i, j)}\right\}$. Thus,

$$
\begin{aligned}
\ln \mathcal{Z}_{0} & =-\frac{1}{2} \operatorname{Tr} \ln \mathcal{G}_{0}-\sum_{n=1}^{\infty} \operatorname{Tr} \ln \mathcal{G}_{n} \\
& =-\frac{1}{2} \sum_{n=-\infty}^{\infty} \operatorname{Tr} \ln \mathcal{G}_{n} .
\end{aligned}
$$

The sum over $n$ can of course be calculated (see for example [20]), so that we may write the corresponding contribution to the free energy as follows:

$$
\mathcal{F}_{0}=\frac{1}{2} \operatorname{Tr} \sqrt{H}+\beta^{-1} \operatorname{Tr} \ln \left(1-e^{-\beta \sqrt{H}}\right)
$$

where an operator $H$ is introduced, such that its matrix elements in the matrix basis $\left\{b^{(i, j)}\right\}$ are

$$
\begin{aligned}
H^{\left(i_{1}, i_{2} ; j_{1}, j_{2}\right)} \equiv & \int d^{2} x\left[b^{\left(i_{1}, i_{2}\right)}(\mathbf{x})\right]^{\dagger} H(\mathbf{x}) b^{\left(j_{1}, j_{2}\right)}(\mathbf{x}) \\
= & \left(m^{2}+\mu^{2}\left(i_{1}+i_{2}+1\right)\right) \delta_{i_{1} j_{1}} \delta_{i_{2} j_{2}} \\
& -\mu^{2} \sqrt{\omega} \sqrt{\left(i_{1}+1\right)\left(i_{2}+1\right)} \delta_{i_{1}+1, j_{1}} \delta_{i_{2}+1, j_{2}} \\
& -\mu^{2} \sqrt{\omega} \sqrt{i_{1} i_{2}} \delta_{i_{1}-1, j_{1}} \delta_{i_{2}-1, j_{2}} .
\end{aligned}
$$

In what follows, the first ( $\beta$-independent) term in (2.36), which corresponds to the vacuum energy part, shall be neglected since it does not contribute to the thermodynamical properties of the system.

We proceed in the next paragraphs to evaluate $\mathcal{F}_{0}$ for different values of the constant $\Omega^{2}$. We first consider the simplest $\Omega^{2}=1$ case, and then extend the result to the general $\Omega^{2} \neq 1$ situation by mapping the latter to the former.

\subsubsection{The self-dual $\Omega^{2}=1$ case}

The $\Omega^{2}=1$ case becomes simple since $\omega$ given by (2.29) becomes zero and therefore the $H$ kernel is diagonal. Explicitly,

$$
H^{\left(i_{1}, i_{2} ; j_{1}, j_{2}\right)}=\left(m^{2}+\frac{4}{\theta}\left(i_{1}+i_{2}+1\right)\right) \delta_{i_{1} j_{1}} \delta_{i_{2} j_{2}} .
$$

It's eigenvalues $h_{l}$ are

$$
h_{l}=\left(m^{2}+\frac{4}{\theta}(l+1)\right), l=0,1,2, \ldots
$$

with a degeneracy equal to $l+1$ (the number of different ways to obtain an integer $l$ by adding two non-negative integers $i_{1}$ and $i_{2}$ ). The degeneracy in the energy for the free theory can be seen to have its origin in the existence of a dynamical SU(2) symmetry for the two dimensional isotropic oscillator (more on this below, see section 2.5). The free energy of the self-dual model is then

$$
\left[\mathcal{F}_{0}\right]_{\Omega^{2}=1}=\beta^{-1} \sum_{l=1}^{\infty} l \ln \left(1-e^{-\beta \sqrt{m^{2}+\frac{4}{\theta} l}}\right) .
$$




\subsubsection{The general case}

The general case corresponding to an arbitrary value of $\Omega^{2}$ can also be dealt with exactly. We first note that the matrix elements of $H$ may be regarded as the ones corresponding to an Hermitian operator constructed out of two independent sets of harmonic oscillator annihilation and creation operators $a_{\alpha}, a_{\alpha}^{\dagger}(\alpha=1,2)$, as follows:

$$
H=m^{2}+\mu^{2}\left(a_{1}^{\dagger} a_{1}+a_{2}^{\dagger} a_{2}+1\right)-\mu^{2} \sqrt{\omega}\left(a_{1} a_{2}+a_{1}^{\dagger} a_{2}^{\dagger}\right) .
$$

The form of (2.41) will be simplified by performing a Bogoliubov transformation. We first introduce a two-component vector a:

$$
\mathbf{a}=\left(\begin{array}{c}
a_{1} \\
a_{2}^{\dagger}
\end{array}\right),
$$

from which a new two-component operator $\mathbf{a}(\alpha)$ is obtained by performing the (unitary) Bogoliubov transformation

$$
\mathbf{a}(\alpha)=U^{\dagger}(\alpha) \mathbf{a} \mathrm{U}(\alpha)
$$

with

$$
\mathrm{U}(\alpha)=e^{i \alpha G}
$$

and the infinitesimal generator $G$ given by

$$
G=i\left(a_{1} a_{2}-a_{2}^{\dagger} a_{1}^{\dagger}\right) \text {. }
$$

The transformation $\mathrm{U}(\alpha)$ maps the original operators $a_{i}$ to new ones $b_{i}$, such that

$$
\mathbf{b} \equiv \mathbf{a}(\alpha)=\left(\begin{array}{c}
\cosh \alpha \sinh \alpha \\
\sinh \alpha \\
\cosh \alpha
\end{array}\right) \mathbf{a} .
$$

We shall fix the hyperbolic angle $\alpha$ by requiring the transformed operator $H(\alpha) \equiv$ $U^{\dagger}(\alpha) H \mathrm{U}(\alpha)$, to be diagonal in terms of the new operators $b_{i}$. This amounts to the equation

$$
\tanh (2 \alpha)=\frac{\Omega^{2}-1}{\Omega^{2}+1},
$$

to be satisfied. In terms of the new operators, the rotated Hamiltonian $H(\alpha)$ adopts the form

$$
\begin{aligned}
H(\alpha) & =m^{2}+\frac{\mu^{2}}{\cosh 2 \alpha}\left(b_{1}^{\dagger} b_{1}+b_{2}^{\dagger} b_{2}+1\right) \\
& =m^{2}+\frac{4 \Omega}{\theta}\left(b_{1}^{\dagger} b_{1}+b_{2}^{\dagger} b_{2}+1\right) .
\end{aligned}
$$

It is then immediate to obtain the free energy in the general case from the one corresponding to the self-dual case by making the substitution $\frac{4}{\theta} \rightarrow \frac{4 \Omega}{\theta}$ in (2.40),

$$
\mathcal{F}_{0}=\beta^{-1} \sum_{l=1}^{\infty} l \ln \left(1-e^{-\beta \sqrt{m^{2}+\frac{4 \Omega}{\theta} l}}\right) .
$$


It is instructive to consider the small $\Omega$ limit of the expression above, since we expect it to be related to the corresponding free energy in the absence of the harmonic $\Omega^{2}$-term. Besides, since this term plays the role of a confining potential, it naturally defines an effective physical volume $V$ of order $\theta /(4 \Omega)$. To simplify matters, we assume $m=0$. For $\Omega \ll 1$, we approximate the sum in (2.49) by an integral, by making an elementary change of variables, one obtains,

$$
\mathcal{F}_{0} \simeq T^{3} V \frac{\theta T^{2}}{4 \Omega} \int_{0}^{\infty} d y y \ln \left(1-e^{-\sqrt{y}}\right)
$$

The free energy density $f_{0}$ is therefore

$$
f_{0} \simeq T^{3} \frac{\theta T^{2}}{4 \Omega} \int_{0}^{\infty} d y y \ln \left(1-e^{-\sqrt{y}}\right)
$$

This should be compared with the free energy density for a free commutative scalar field in a box (which coincides with the result for a free noncommutative theory in an infinite volume with no harmonic term)

$$
\left[f_{0}\right]_{\text {comm }}=T^{3} \frac{1}{2 \pi} \int_{0}^{\infty} d y y \ln \left(1-e^{-y}\right) .
$$

An important qualitative difference between the results (2.51) and (2.52) can be noted due to the appearance of a (dimensionless) factor $\frac{\pi \theta T^{2}}{2 \Omega}$.

The $\Omega \rightarrow 0$ limit depends then on whether one takes it before or after evaluating the free energy. Indeed, a free NCQFT without the GW term $(\Omega=0)$ yields (2.52), which coincides with the result for a free commutative QFT. On the other hand, we see that taking the $\Omega \rightarrow 0$ limit after the evaluation of the free energy yields instead a divergent result and does not match the $\Omega=0$ result. This behavior is reminiscent to the well known subtlety when taking the commutative $\theta \rightarrow 0$ limit of non-commutative theories [13].

We conclude this subsection by noting that the transformation that diagonalices $H$ is of the type considered when dealing with 'two-mode squeezed states', in a quite different context [21]. We can however, take advantage of the equivalent form of the transformation in order to extract conclusions for the case at hand.

As an example, the ground state of the model for arbitrary $\Omega$ may be obtained as follows: since the transformed operator $H(\alpha)$ in (2.48) is diagonal in the $b_{i}$ basis, we may read off its 'ground state' $|0(\alpha)\rangle$ as the one annihilated by the $b_{i}$ operators. Taking advantage of the relation (2.46) it is therefore possible to write an explicit relation between the ground state for arbitrary $\Omega,|0\rangle_{\Omega} \equiv|0(\alpha)\rangle$, and the ground state $|0\rangle_{\Omega=1} \equiv|0\rangle$ of the self-dual Hamiltonian (2.38). The relation between them can be shown to be given by [21],

$$
|0(\alpha)\rangle=\frac{1}{\cosh \alpha} e^{-\tanh \alpha a_{1}^{\dagger} a_{2}^{\dagger}}|0\rangle .
$$

Or, in terms of the model parameters,

$$
|0\rangle_{\Omega}=\frac{2 \sqrt{\Omega}}{1+\Omega} e^{-\left|\frac{1-\Omega}{1+\Omega}\right| a_{1}^{\dagger} a_{2}^{\dagger}}|0\rangle_{\Omega=1} .
$$

This is an example of a two-mode squeezed state (see [21]). 


\subsection{First-order term}

We proceed now to evaluate the first-order contribution to the free energy, $\mathcal{F}_{I}^{(1)}$. The expression for (2.10) in terms of the (Matsubara) Fourier components for the field $\phi$ is

$$
\mathcal{F}_{I}^{(1)}=\frac{2 \pi \lambda \theta}{4 ! \beta^{2}} \sum_{n_{1}, \ldots, n_{4}=-\infty}^{+\infty} \sum_{i_{1}, \ldots, i_{4}=0}^{+\infty} \delta_{\sum_{i} n_{i}=0}\left\langle\phi_{n_{1}}^{\left(i_{1}, i_{2}\right)} \phi_{n_{2}}^{\left(i_{2}, i_{3}\right)} \phi_{n_{3}}^{\left(i_{3}, i_{4}\right)} \phi_{n_{4}}^{\left(i_{4}, i_{1}\right)}\right\rangle
$$

Moreover, since the averages above are defined by a quadratic weight, an application of Wick's theorem yields,

$$
\begin{aligned}
\left\langle\phi_{n_{1}}^{\left(i_{1}, i_{2}\right)} \phi_{n_{2}}^{\left(i_{2}, i_{3}\right)} \phi_{n_{3}}^{\left(i_{3}, i_{4}\right)} \phi_{n_{4}}^{\left(i_{4}, i_{1}\right)}\right\rangle= & \left\langle\phi_{n_{1}}^{\left(i_{1}, i_{2}\right)} \phi_{n_{2}}^{\left(i_{2}, i_{3}\right)}\right\rangle\left\langle\phi_{n_{3}}^{\left(i_{3}, i_{4}\right)} \phi_{n_{4}}^{\left(i_{4}, i_{1}\right)}\right\rangle \\
& +\left\langle\phi_{n_{1}}^{\left(i_{1}, i_{2}\right)} \phi_{n_{3}}^{\left(i_{3}, i_{4}\right)}\right\rangle\left\langle\phi_{n_{2}}^{\left(i_{2}, i_{3}\right)} \phi_{n_{4}}^{\left(i_{4}, i_{1}\right)}\right\rangle \\
& +\left\langle\phi_{n_{1}}^{\left(i_{1}, i_{2}\right)} \phi_{n_{4}}^{\left(i_{4}, i_{1}\right)}\right\rangle\left\langle\phi_{n_{2}}^{\left(i_{2}, i_{3}\right)} \phi_{n_{3}}^{\left(i_{3}, i_{4}\right)}\right\rangle .
\end{aligned}
$$

Since the quadratic action $S_{0}$ only mixes Fourier components such that their Matsubara indices add up to zero, we have,

$$
\begin{aligned}
\mathcal{F}_{I}^{(1)}=\frac{2 \pi \lambda \theta}{4 ! \beta^{2}} & \sum_{n_{1}, n_{2}} \sum_{i_{1}, \ldots, i_{4}}\left(\left\langle\phi_{-n_{1}}^{\left(i_{1}, i_{2}\right)} \phi_{n_{1}}^{\left(i_{2}, i_{3}\right)}\right\rangle\left\langle\phi_{-n_{2}}^{\left(i_{3}, i_{4}\right)} \phi_{n_{2}}^{\left(i_{4}, i_{1}\right)}\right\rangle\right. \\
& \left.+\left\langle\phi_{-n_{1}}^{\left(i_{1}, i_{2}\right)} \phi_{n_{1}}^{\left(i_{3}, i_{4}\right)}\right\rangle\left\langle\phi_{-n_{2}}^{\left(i_{2}, i_{3}\right)} \phi_{n_{2}}^{\left(i_{4}, i_{1}\right)}\right\rangle+\left\langle\phi_{-n_{1}}^{\left(i_{1}, i_{2}\right)} \phi_{n_{1}}^{\left(i_{4}, i_{1}\right)}\right\rangle\left\langle\phi_{-n_{2}}^{\left(i_{2}, i_{3}\right)} \phi_{n_{2}}^{\left(i_{3}, i_{4}\right)}\right\rangle\right)
\end{aligned}
$$

Relabeling indices and combining identical terms we end up with,

$$
\begin{aligned}
\mathcal{F}_{I}^{(1)}=\frac{2 \pi \lambda \theta}{4 ! \beta^{2}} \sum_{n_{1}, n_{2}} \sum_{i_{1}, \ldots, i_{4}}( & 2\left\langle\phi_{-n_{1}}^{\left(i_{1}, i_{2}\right)} \phi_{n_{1}}^{\left(i_{2}, i_{3}\right)}\right\rangle\left\langle\phi_{-n_{2}}^{\left(i_{3}, i_{4}\right)} \phi_{n_{2}}^{\left(i_{4}, i_{1}\right)}\right\rangle \\
& \left.+\left\langle\phi_{-n_{1}}^{\left(i_{1}, i_{2}\right)} \phi_{n_{1}}^{\left(i_{3}, i_{4}\right)}\right\rangle\left\langle\phi_{-n_{2}}^{\left(i_{2}, i_{3}\right)} \phi_{n_{2}}^{\left(i_{4}, i_{1}\right)}\right\rangle\right) .
\end{aligned}
$$

The first term can be interpreted as a planar graph in standard double line notation (see [13]), while the second one can be seen to be non-planar. In the expressions above, the two point free correlation function is determined from the quadratic action (2.13), and its form strongly depends on the value of $\Omega$.

As we did for the zero-order term, we perform in the following section the explicit calculation for the self-dual point. We shall then comment on the general case computation.

\subsubsection{Self-dual case}

As discussed in section 2.3.1 the $\Omega=1$ case is particularly simple since the quadratic part of the action is diagonal. We now proceed to compute the first-order term (2.58) taking into account that the two point correlation function adopts, for the self-dual case, the quite simple form,

$$
\begin{aligned}
\left\langle\phi_{-n}^{\left(i_{1}, i_{2}\right)} \phi_{n}^{\left(j_{1}, j_{2}\right)}\right\rangle & =\left\langle\bar{\phi}_{n}^{\left(i_{2}, i_{1}\right)} \phi_{n}^{\left(j_{1}, j_{2}\right)}\right\rangle \\
& =\frac{1}{2 \pi \theta}\left(m^{2}+\omega_{n}^{2}+\frac{4}{\theta}\left(i_{1}+i_{2}+1\right)\right)^{-1} \delta_{i_{1} j_{2}} \delta_{i_{2} j_{1}} .
\end{aligned}
$$


We separate the planar and non-planar contributions as,

$$
\mathcal{F}_{I}^{(1)}=P+Q
$$

where

$$
\begin{aligned}
P=\frac{\lambda}{4 ! \beta^{2} \pi \theta} \sum_{n_{1}, n_{2}} \sum_{i_{1}, i_{2}, i} & {\left[\left(m^{2}+\omega_{n_{1}}^{2}+\frac{4}{\theta}\left(i_{1}+i+1\right)\right)^{-1}\right.} \\
& \left.\times\left(m^{2}+\omega_{n_{2}}^{2}+\frac{4}{\theta}\left(i_{2}+i+1\right)\right)^{-1}\right],
\end{aligned}
$$

and

$$
\begin{aligned}
Q=\frac{\lambda}{4 ! 2 \pi \theta \beta^{2}} \sum_{n_{1}, n_{2}} \sum_{i} & {\left[\left(m^{2}+\omega_{n_{1}}^{2}+\frac{4}{\theta}(2 i+1)\right)^{-1}\right.} \\
& \left.\times\left(m^{2}+\omega_{n_{2}}^{2}+\frac{4}{\theta}(2 i+1)\right)^{-1}\right] .
\end{aligned}
$$

The $i$ 's indices structure in the last two equations manifest the expected worse UV behavior for the planar contribution (2.61) as compared to the non-planar one (2.62). ${ }^{4}$

It is useful for what follows to simplify the expressions (2.61)-(2.62). We first note that the planar contribution may be written as,

$$
P=\frac{\lambda}{4 ! \pi \theta} \sum_{i=0}^{\infty}[\mathcal{S}(i)]^{2}
$$

where

$$
\mathcal{S}(i)=\frac{1}{\beta} \sum_{n=-\infty}^{\infty} \sum_{j=0}^{\infty}\left(m^{2}+\omega_{n}^{2}+\frac{4}{\theta}(i+j+1)\right)^{-1} .
$$

For the non-planar term, we have instead,

$$
Q=\frac{\lambda}{4 ! 2 \pi \theta} \sum_{i=0}^{\infty}[\mathcal{T}(i)]^{2},
$$

where

$$
\mathcal{T}(i)=\frac{1}{\beta} \sum_{n=-\infty}^{\infty}\left(m^{2}+\omega_{n}^{2}+\frac{4}{\theta}(2 i+1)\right)^{-1} .
$$

It is evident that there are UV divergences lurking in the expressions (2.63)-(2.65), and we now deal with them. We will show below that to first order in $\lambda$, as it happens in the commutative case at finite temperature (see [18]), only a mass counterterm is required to give meaning to the free energy.

Let us begin computing the two point function counterterm to first order in $\lambda$, since it should be taken into account in the calculation of the free energy we performed above.

\footnotetext{
${ }^{4}$ The number of sums in $i$ for any diagram can be seen to be equal, when drawing it in double line notation, to the number of independent loops.
} 
It is straightforward to see that the divergent contribution to the quadratic part of the effective action comes from a planar tadpole diagram and takes the form,

$$
\left.\Gamma_{2}[\phi]\right|_{\text {div }}=\frac{\lambda}{3 !(2 \pi \theta)} \mathcal{S}_{0}\left(i_{s}\right) \int_{0}^{\beta} d \tau \int d^{2} x \phi \star \phi,
$$

where $\mathcal{S}_{0}(i)$ can be identified as the zero temperature part of $\mathcal{S}(i)$, namely,

$$
\mathcal{S}_{0}(i)=\int \frac{d \omega}{2 \pi} \sum_{k=0}^{\infty} \frac{1}{\omega^{2}+m^{2}+\frac{4}{\theta}(k+i+1)},
$$

easily seen to be UV divergent for any arbitrary integer $i$ (interpreted in (2.67) as the substraction point). Performing the integral over $\omega$, one can write this last expression as,

$$
\mathcal{S}_{0}(i)=\frac{\sqrt{\theta}}{4} \zeta\left(\frac{1}{2}, \frac{m^{2} \theta}{4}+i+1\right)
$$

where now, $\zeta(s, a)$ should be understood as the analytical continuation in $s$ of the generalized Riemann (Hurwitz) $\zeta$ function. The analytical regularization renders a finite result for (2.69), the (infinite) ambiguity constant of which is fixed by a renormalization condition for the mass parameter.

We therefore fix the mass counterterm to have the form, ${ }^{5}$

$$
\delta m^{2}=-\frac{\lambda}{3 ! \pi \theta} \mathcal{S}_{0}(0) .
$$

This counterterm cancels the relevant divergences in $P$ when the regularization is removed. Separating $\mathcal{S}(i)$ into its zero temperature part $\mathcal{S}_{0}$ plus its thermal contribution $\mathcal{S}_{T}$ (which vanishes at $T=0$ ), we get,

$$
\begin{aligned}
P= & \frac{\lambda}{4 ! \pi \theta} \sum_{i=0}^{\infty} \mathcal{S}_{T}(i) \mathcal{S}_{T}(i) \\
& +\frac{2 \lambda}{4 ! \pi \theta} \sum_{i=0}^{\infty} \mathcal{S}_{0}(i) \mathcal{S}_{T}(i)+\frac{\lambda}{4 ! \pi \theta} \sum_{i=0}^{\infty} \mathcal{S}_{0}(i) \mathcal{S}_{0}(i)
\end{aligned}
$$

The last term will be ignored from now on, since we are not interested in temperatureindependent terms (which amount to vacuum energy contributions). The divergence in the second term is, on the other hand, exactly canceled by the chosen mass counterterm (2.70). Thus, the renormalized $P$ becomes,

$$
P_{\text {ren }}=\frac{\lambda}{4 ! \pi \theta} \sum_{i=0}^{\infty} \mathcal{S}_{T}(i) \mathcal{S}_{T}(i)+\frac{2 \lambda}{4 ! \pi \theta} \sum_{i=0}^{\infty} \tilde{\mathcal{S}}_{0}(i) \mathcal{S}_{T}(i)
$$

where $\tilde{\mathcal{S}}_{0}(i) \equiv \mathcal{S}_{0}(i)-\mathcal{S}_{0}(0)$.

\footnotetext{
${ }^{5}$ We fix the substraction point $i_{s}$ in $(2.67)$ to be zero.
} 
On the other hand, the divergent part of $Q$ gets canceled by just subtracting the temperature independent (vacuum-energy) contribution. Indeed, splitting $\mathcal{T}$ as we did for $\mathcal{S}$, we have,

$$
Q_{\text {ren }}=\frac{\lambda}{4 ! 2 \pi \theta} \sum_{i=0}^{\infty} \mathcal{T}_{T}(i) \mathcal{T}_{T}(i)+\frac{2 \lambda}{4 ! 2 \pi \theta} \sum_{i=0}^{\infty} \mathcal{T}_{0}(i) \mathcal{T}_{T}(i),
$$

where (contrary to what happened in the calculation of $P_{\text {ren }}$ ) there is no contribution to $Q$ from the counterterm (2.70), since its insertion yields, to this order, only planar contributions.

Explicitly the function $\tilde{\mathcal{S}}_{0}(i)$ takes the form,

$$
\begin{aligned}
\tilde{\mathcal{S}}_{0}(i) & =\frac{\sqrt{\theta}}{4}\left[\zeta\left(\frac{1}{2}, \frac{m^{2} \theta}{4}+i+1\right)-\zeta\left(\frac{1}{2}, \frac{m^{2} \theta}{4}+1\right)\right] \\
& =-\frac{\sqrt{\theta}}{4} \sum_{k=1}^{i} \frac{1}{\sqrt{k+\frac{m^{2} \theta}{4}}} .
\end{aligned}
$$

The temperature dependent piece, $\mathcal{S}_{T}(i)$ is, on the other hand, given by

$$
\begin{aligned}
\mathcal{S}_{T}(i) & =\frac{\sqrt{\theta}}{2} \sum_{j=0}^{\infty} \frac{1}{\sqrt{\frac{m^{2} \theta}{4}+j+i+1}} \frac{1}{e^{\frac{2 \beta}{\sqrt{\theta}} \sqrt{\frac{m^{2} \theta}{4}+j+i+1}}-1} \\
& =\frac{\sqrt{\theta}}{2} \sum_{j=i}^{\infty} \frac{1}{\sqrt{\frac{m^{2} \theta}{4}+j+1}} \frac{1}{e^{\frac{2 \beta}{\sqrt{\theta}} \sqrt{\frac{m^{2} \theta}{4}+j+1}}-1} .
\end{aligned}
$$

For $\mathcal{T}(i)$ we obtain,

$$
\mathcal{T}_{0}(i)=\frac{\sqrt{\theta}}{4} \frac{1}{\sqrt{\frac{m^{2} \theta}{4}+2 i+1}}
$$

and

$$
\mathcal{T}_{T}(i)=\frac{\sqrt{\theta}}{2} \frac{1}{\sqrt{\frac{m^{2} \theta}{4}+2 i+1}} \frac{1}{e^{\frac{2 \beta}{\sqrt{\theta}} \sqrt{\frac{m^{2} \theta}{4}+2 i+1}}-1} .
$$

Inserting the previous expressions into $P_{\text {ren }}$ and $Q_{\text {ren }}$ yields the first order contribution in $\lambda$ to the free energy as a combination of multiple series, which cannot, in general, be summed in closed form. However, for the massless case it is easy to see that the first order correction will have the form

$$
\mathcal{F}_{I}^{(1)}=\lambda f(\sqrt{\theta} T) .
$$

This is, $\lambda$ times a function of the dimensionless combination involving the noncommutativity parameter $\theta$ and the temperature $T$. In the limit $\sqrt{\theta} T \gg 1$, one can also see that the leading behavior of the free energy is of the form $f(x) \sim x^{2}$, thus,

$$
\mathcal{F}_{I}^{(1)} \sim \lambda \theta T^{2}
$$

This leading contribution comes only from the planar diagrams $P$, as the non-planar ones $Q$ vanish in this limit. 
It is worth noting that, in the massless limit, the corresponding contribution for the commutative analogue of this model has a similar form,

$$
\left[\mathcal{F}_{I}^{(1)}\right]_{\mathrm{comm}}=\kappa \lambda L^{2} T^{2}
$$

where $L^{2}$ is the 'volume' (i.e., area) of the system, and $\kappa$ is a numerical constant. The noncommutativity parameter $\theta$ appears again as playing the role of the area of the system, at least when that parameter is big in comparison with the temperature. This is our second argument for interpreting $\frac{\theta}{4 \Omega}$ (here $\Omega=1$ ) as the 'volumen' of the system.

\subsubsection{General case}

For general $\Omega$ the complicated form of the propagator renders the calculation of first order computations quite involved. However, when considering the free energy in the large $\theta$ ('thermodynamic') limit, an important simplification arises. Indeed, in this limit only the planar diagrams contribute: the reason (as it happened for the self-dual case) is that non-planar graphs, having less independent summations, have a softer scaling behavior in the thermodynamic limit, and therefore get suppressed. Since only planar diagrams are relevant, one can then take advantage of the Bogoliubov transformation of the free case, which allows one to map the non self-dual case to the dual one, by a simple rescaling of $\theta$ in the propagators. For the planar contribution (and only for them) the unitary operators cancel out. Thus, in the thermodynamic limit, the result for the free energy correction $\mathcal{F}_{I}^{(1)}$ only differ from a $\frac{1}{\Omega}$ factor in comparison with the self-dual contribution (as it was the case for the ideal gas term).

\subsection{Summation of the planar ring diagrams for the self-dual case}

We conclude this section by considering the summation of the planar ring diagrams (non planar are discarded, since we have in mind the large-volume limit). We do this in order to exhibit some of the peculiarities of the present model.

The second order term in the (renormalized) effective action is given by,

$$
\begin{aligned}
{\left[\Gamma_{2}(\phi)\right]_{\mathrm{ren}}=\frac{\lambda}{2 \times 3 !} \int_{0}^{\beta} d \tau \sum_{i, j=0}^{\infty} \phi^{(j, i)}(\tau)\left[\tilde{\mathcal{S}}_{0}(i)+\mathcal{S}_{T}(i)\right.} \\
\left.+\tilde{\mathcal{S}}_{0}(j)+\mathcal{S}_{T}(j)\right] \phi^{(i, j)}(\tau),
\end{aligned}
$$

where we have kept the same notations as in the previous subsection.

We note that, being this contribution diagonal in the matrix base, the contribution corresponding to the summation of the ring diagrams can be written straightforwardly. Indeed, it corresponds to the calculation of the Gaussian functional integral that results from the inclusion of the quadratic term in the effective action, and subtracting the (already written) lower-order terms, to avoid double counting. The expression can be put in the 
following way,

$$
\begin{aligned}
\mathcal{F}_{\text {ring }}= & \frac{1}{2 \beta} \sum_{n=-\infty}^{\infty} \sum_{i, j=0}^{\infty} \ln \left\{\omega_{n}^{2}+m^{2}+\frac{4}{\theta}(i+j+1)\right. \\
& \left.+\frac{\lambda}{3 ! 2 \pi \theta}\left[\tilde{\mathcal{S}}_{0}(i)+\mathcal{S}_{T}(i)+\tilde{\mathcal{S}}_{0}(j)+\mathcal{S}_{T}(j)\right]\right\} \\
& -\mathcal{F}_{0}-P .
\end{aligned}
$$

Note that, for $n=0$ and $m=0$, the would-be IR divergent contributions are not only cured by the first order correction self-energy term, but also by the $\theta^{-1}$ factor always present whenever there is a finite volume, whose origin is the zero point energy of the two dimensional oscillators. The summation over the Matsubara frequencies can be performed leading to,

$$
\mathcal{F}_{\text {ring }}=\beta^{-1} \sum_{i, j=0}^{\infty} e^{-\beta \sqrt{m^{2}+\mathcal{E}_{i, j}}}-\mathcal{F}_{0}-P
$$

where

$$
\mathcal{E}_{i, j}=\frac{4}{\theta}(i+j+1)+\frac{\lambda}{3 ! 2 \pi \theta}\left[\tilde{\mathcal{S}}_{0}(i)+\mathcal{S}_{T}(i)+\tilde{\mathcal{S}}_{0}(j)+\mathcal{S}_{T}(j)\right] .
$$

We conclude by mentioning an important outcome of this expression: since each element in the sum is no longer a function of $i+j$, the degeneracy we had for the free case is lifted. This may be thought of as due to the fact that, when including the $\phi_{\star}^{4}$ interaction, the dynamical SU(2) symmetry of the free theory cannot be preserved.

\section{Conclusions}

We have considered the perturbative calculation of the free energy for a noncommutative real scalar field theory in $2+1$ dimensions in the presence of a Grosse-Wulkenhaar term. We have first shown, at the free ('ideal gas') level, that the free energy for the GW-model has a qualitatively different temperature behavior when compared to the known result obtained by assuming the noncommutative theory to be defined on an infinite volume from the very beginning. The qualitative difference being due to the appearance of the dimensionless parameter $\frac{\theta T^{2}}{4 \Omega}$ in (2.51). Moreover, for the known infinite volume $(\Omega=0)$ case, the free energy turns out to be $\theta$-independent since the $\theta$-dependence can solely arise from boundary terms which vanish for our choice of boundary conditions.

Of course, one might have also considered the $\Omega=0$ case in a finite-volume situation. That procedure should also produce, we believe, a non trivial large-volume limit, due to the interplay between the noncommutativity and boundary conditions.

Regarding the perturbative corrections, in spite of the difficulties to obtain analytical results, some general properties clearly emerge. Firstly, the perturbative computations and the harmonic potential form of the GW-term suggest to interpret the volume of the system to be given by $V \sim \theta / 4 \Omega$. Secondly, in the thermodynamic $\theta \rightarrow \infty$ limit, only planar graphs yield a non-vanishing contribution. Moreover, for the arbitrary $\Omega$ case, one can see that, again in the thermodynamic limit, the contribution of the planar graphs, can 
be obtained from the calculation of the ones for the self-dual case, by a redefinition of the propagator, which essentially amounts to a rescaling of $\theta$.

Finally, we have constructed a series that represent the sum of the planar ring diagrams, showing how the GW-term moderates its IR behavior. As an outcome, the calculation shows that the dynamical $\mathrm{SU}(2)$ symmetry present in the free theory is not preserved when interactions are turned on.

The renormalization process that gives meaning to the perturbative computations goes in complete analogy with the commutative case. We have shown that, as in the commutative case, at the first perturbative order no new divergences appear at finite temperature, and the expressions get regularized, if the divergences at zero temperature where already tamed. We should mention nevertheless, that contrary to the standard $\Omega=0$ case, the planar tadpole contributions to the self energy depend on the external momentum.

\section{Acknowledgments}

G.A.S. would like to thank Glenn Barnich and Andy Gomberoff for email correspondence. C.D.F. thanks CONICET and ANPCyT for financial support and G.A.S. acknowledges support from CONICET, PIP 6160.

\section{A. Useful formulae and Feynman diagrams conventions}

\section{Conventions}

Noncommutativity affects the two spatial coordinates $x_{i}(i, j=1,2)$,

$$
\left[x_{0}, x_{i}\right]=0,\left[x_{i}, x_{j}\right]=i \theta \epsilon_{i j},
$$

and is realized in terms of the star product (1.1).

The Moyal-Weyl correspondence [2, 22 maps integration on NC space to traces on Fock space as

$$
\int d^{2} x f(x)=2 \pi \theta \operatorname{Tr}\left[\mathcal{O}_{f}\right]
$$

The association between functions $f(\mathbf{x})$ in $\mathrm{NC}$ space and Weyl ordered operators $\mathcal{O}_{f}$ is via

$$
\mathcal{O}_{f}(\hat{\mathbf{x}})=\int d^{2} x f(\mathbf{x}) \hat{\Delta}(\mathbf{x})
$$

where

$$
\hat{\Delta}(\mathbf{x})=\int \frac{d^{2} k}{(2 \pi)^{2}} e^{i \mathbf{k} \cdot(\hat{\mathbf{x}}-\mathbf{x})}
$$

One can see that derivatives on $\mathrm{NC}$ space can be implemented as

$$
\partial_{i} f=\frac{i}{\theta} \epsilon_{i j}\left[x_{j}, f\right]
$$

The matrix base functions $b^{(i, j)}(\mathbf{x})$ appearing in the text are the Weyl ordered representation in NC space of the Fock space operators $|i\rangle\langle j|$. One therefore has $\left[b^{(i, j)}(\mathbf{x})\right]^{\dagger}=b^{(j, i)}(\mathbf{x})$. 


\section{On finite temperature energy sums}

To separate the temperature dependence from the zero temperature contribution in expressions (2.64) and (2.66) we used the identity

$$
\sum_{n=-\infty}^{\infty} \frac{1}{n^{2}+a^{2}}=\frac{2 \pi}{a}\left[\frac{1}{2}+\frac{1}{e^{2 \pi a}-1}\right]
$$

\section{Diagrammatics}

To keep track of the matrix indices of the field in (2.22) a double line notation is useful. The free theory two point propagator $(2.59)$ can be drawn as

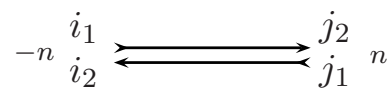

The fourth order vertex (2.14) in matrix base (2.55) is represented as

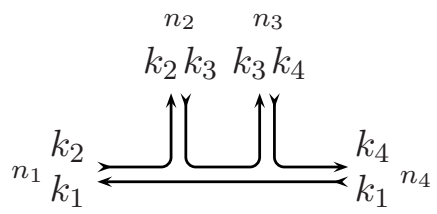

The first order corrections to the free energy (2.60) are
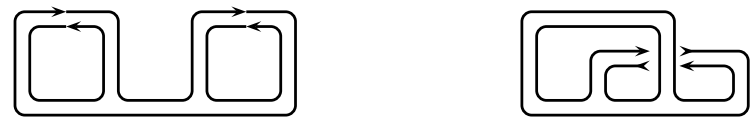

\section{Propagator corrections and self-energy}

The two point propagator function is defined as

$$
D\left(\mathbf{x}_{1}, t_{1} \mid \mathbf{x}_{2}, t_{2}\right)=\left\langle\phi\left(\mathbf{x}_{1}, t_{1}\right) \phi\left(\mathbf{x}_{2}, t_{2}\right)\right\rangle .
$$

Translational invariance in $t$ coordinate implies that $D$ is a function of $t_{1}-t_{2}$. The kernel $\tilde{D}_{n}$ in Fourier space is defined by (see (2.17))

$$
D\left(\mathbf{x}_{1}, t_{1} \mid \mathbf{x}_{2}, t_{2}\right)=\frac{1}{\beta} \sum_{n=-\infty}^{\infty} \sum_{i, j=0}^{\infty} e^{i \omega_{n}\left(t_{1}-t_{2}\right)} b^{\left(i_{1}, j_{1}\right)}\left(\mathbf{x}_{1}\right) b^{\left(i_{2}, j_{2}\right)}\left(\mathbf{x}_{2}\right) \tilde{D}_{n}^{\left(i_{1}, j_{1} ; i_{2}, j_{2}\right)}
$$

where $\omega_{n}$ are the Matsubara frequencies, and takes the form

$$
\tilde{D}_{n}^{\left(i_{1}, j_{1} ; i_{2}, j_{2}\right)}=\left\langle\phi_{-n}^{\left(i_{1}, j_{1}\right)} \phi_{n}^{\left(i_{2}, j_{2}\right)}\right\rangle
$$

The first order corrections to the propagator in a diagrammatic expansion are

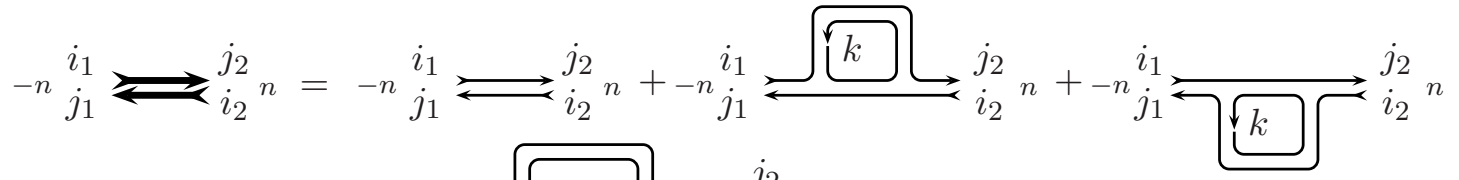

$$
\begin{aligned}
& + { } _ { - n } i _ { j _ { 1 } } \rightleftharpoons \longdiv { \rightleftarrows } \rightleftarrows \begin{array} { c } 
{ j _ { 2 } } \\
{ i _ { 2 } { } ^ { n } }
\end{array}
\end{aligned}
$$




\section{References}

[1] M.R. Douglas and N.A. Nekrasov, Noncommutative field theory, Rev. Mod. Phys. 73 (2001) 977 hep-th/0106048.

[2] R.J. Szabo, Quantum field theory on noncommutative spaces, Phys. Rept. 378 (2003) 207 hep-th/0109162.

[3] N. Seiberg and E. Witten, String theory and noncommutative geometry, JHEP 09 (1999) 032 hep-th/9908142.

[4] L. Susskind, The quantum Hall fluid and non-commutative Chern-Simons theory, hep-th/0101029.

[5] C. Duval and P.A. Horvathy, The "Peierls substitution" and the exotic Galilei group, Phys. Lett. B 479 (2000) 284 hep-th/0002233.

[6] W.H. Huang, High temperature effective potential of noncommutative scalar field theory: reduction of degree of freedom by noncommutativity, Phys. Rev. D 63 (2001) 125004 hep-th/0101040.

[7] F.T. Brandt, J. Frenkel and C. Muramoto, On the free energy of noncommutative quantum electrodynamics at high temperature, Nucl. Phys. B 754 (2006) 146 hep-th/0605240.

[8] F.T. Brandt, J. Frenkel and C. Muramoto, Fermionic contributions to the free energy of noncommutative quantum electrodynamics at high temperature, Phys. Lett. B 649 (2007) 483 hep-th/0703242.

[9] A.V. Strelchenko and D.V. Vassilevich, On space-time noncommutative theories at finite temperature, Phys. Rev. D 76 (2007) 065014 arXiv:0705.4294.

[10] H. Grosse and R. Wulkenhaar, Renormalisation of $\phi^{4}$ theory on noncommutative $R^{2}$ in the matrix base, JHEP 12 (2003) 019 hep-th/0307017.

[11] H. Grosse and R. Wulkenhaar, Renormalisation of $\phi^{4}$ theory on noncommutative $R^{4}$ in the matrix base, Commun. Math. Phys. 256 (2005) 305 hep-th/0401128.

[12] V. Rivasseau, Non-commutative renormalization, arXiv:0705.0705.

[13] S. Minwalla, M. Van Raamsdonk and N. Seiberg, Noncommutative perturbative dynamics, JHEP 02 (2000) 020 hep-th/9912072.

[14] E. Langmann and R.J. Szabo, Duality in scalar field theory on noncommutative phase spaces, Phys. Lett. B 533 (2002) 168 hep-th/0202039.

[15] V.P. Nair and A.P. Polychronakos, Quantum mechanics on the noncommutative plane and sphere, Phys. Lett. B 505 (2001) 267 hep-th/0011172.

[16] S. Bellucci, A. Nersessian and C. Sochichiu, Two phases of the non-commutative quantum mechanics, Phys. Lett. B 522 (2001) 345 hep-th/0106138.

[17] E. Langmann, R.J. Szabo and K. Zarembo, Exact solution of quantum field theory on noncommutative phase spaces, JHEP 01 (2004) 017 hep-th/0308043.

[18] J.I. Kapusta and C. Gale, Finite-temperature field theory: principles and applications, Cambridge University Press, Cambridge U.K. (2006).

[19] J.M. Gracia-Bondia and J.C. Varilly, Algebras of distributions suitable for phase space quantum mechanics. 1, J. Math. Phys. 29 (1988) 869. 
[20] H. Kleinert, Path integrals in quantum mechanics, statistics, polymer physics, and financial markets, World Scientific, Singapore (2004).

[21] H. Umezawa, Advanced field theory: micro, macro, and thermal physics, AIP, New York U.S.A. (1993).

[22] J.A. Harvey, Komaba lectures on noncommutative solitons and D-branes, hep-th/0102076. 